Psychotherapeut 2021 $66: 203-208$ https://doi.org/10.1007/s00278-021-00498-2 Angenommen: 11. Januar 2021 Online publiziert: 22. Februar 2021 (c) Springer Medizin Verlag GmbH, ein Teil von Springer Nature 2021

\section{Die Coronapandemie stellt psycho- logisch gesprochen eine abstrakte, unsichtbare Bedrohung dar, die für viele Menschen schwer zu verstehen ist. Die Verschwörungserzählung dagegen strukturiert die Welt. Der Glaube an Verschwörungen ist al- lerdings weder neu noch ein reines Produkt von sozialen Medien. Zwei zentrale Herausforderungen, die sich ergeben, sind, wie man Ver- schwörungserzählungen in der Öffentlichkeit begegnen kann, und wie man im persönlichen oder im therapeutischen Setting mit ihren Anhänger:innen umgeht. Um diesen begegnen zu können, müssen die Mechanismen von Verschwörungs- theorien verstanden werden.}

\section{Hintergrund}

Als die chinesische Gesundheitskommission am 20.01.2020 bekannt gab, dass es eine Mensch-zu-Mensch-Übertragung mit einem neuartigen Coronavirus gegeben habe, war es für viele Menschen noch unvorstellbar, wie stark dieses Virus die eigene Lebenswirklichkeit zumindest temporär verändern würde. Die durch die „Coronavirus-Krankheit 2019“ (COVID-19) ausgelöste Pandemie wirkt sich unterschiedlich auf Menschen aus: Während Infizierte und Erkrankte als primär Betroffene gelten können, gibt es große Teile sekundär Betroffener, die unter den infektionseindämmenden Maßnahmen leiden - weil wichtige medizinische Eingriffe verzögert werden, sie ökonomische Einbußen haben oder mit der Einsamkeit aufgrund sozialer Distanz umgehen müssen.

Darüber sind aber auch Schäden zu beklagen durch die Verbreitung von

Pia Lamberty · Roland Imhoff

Sozial- und Rechtspsychologie, Johannes-Gutenberg-Universität Mainz, Mainz, Deutschland

\title{
Verschwörungserzählungen im Kontext der Coronapandemie
}

Falschinformationen in der Pandemie: Bereits im Januar warnte die Weltgesundheitsorganisation (WHO), dass wir es nicht nur mit einer Pandemie, sondern auch mit einer sogenannten Infodemie $\mathrm{zu}$ tun hätten - und dass diese genauso gefährlich sei wie die Pandemie selbst. Eine Infodemie führt potenziell nicht nur zu einer Polarisierung der öffentlichen Debatte und verstärkt Hassrede, sondern kann auch Leben kosten (WHO 2020). Und eine Infodemie geht auch oft mit dem Verschwörungsglauben einher. Verschwörungsgläubige tendieren eher dazu, sich und andere gesundheitlichen Gefahren auszusetzen. Auch die Verbreitung von antisemitischen und antidemokratischen Inhalten findet sich in dieser Szene immer wieder (Imhoff und Bruder 2014; Imhoff et al. 2021).

Daneben sind es v. a. Angehörige, die nicht verstehen, warum ihnen wichtige Menschen auf einmal anscheinend realitätsfernen Ideen anhängen. Im Folgenden wird erläutert, ob und, wenn ja, warum Verschwörungsnarrative in gesellschaftlichen Krisen wie einer Pandemie Hochkonjunkturen erfahren, welches Weltbild sich in ihnen manifestiert, wer besonders anfällig ist, und welche Konsequenzen sie haben. Abschließend werden Implikationen für die Praxis diskutiert.

\section{Begriffsentwirrung: eine Frage der Mentalität?}

Den Begriff „Verschwörungstheorie“ ist seit den 1860er-Jahren bekannt. Er bezieht sich auf die Annahme, dass als mächtig wahrgenommene Personen oder Gruppen im Geheimen einen Plan schmieden würden, der Gesellschaft zu schaden (Goertzel 1994). In den letzten
Jahren gab es v. a. im deutschsprachigen Raum eine immer stärkere Diskussion darüber, dass dieser Begriff problematisch sei. In der Psychologie wird häufig von der Verschwörungsmentalität gesprochen (Imhoff und Bruder 2014).

Die abstrakte Verschwörungsmentalität spiegelt dabei die generelle Tendenz wider, an Verschwörungen zu glauben. Diese Idee, dass es eine kohärente Verschwörungsmentalität gibt, basiert auf dem wiederholt beobachteten Muster, dass die Zustimmungen $\mathrm{zu}$ inhaltlich sehr divergierenden Ausprägungen des Verschwörungsglaubens stets positiv miteinander korrelieren und auf einem Faktor laden. Als vielleicht plakativstes Beispiel zeigte sich in den Studien von Wood et al. (2012), dass wer glaubt, dass Lady Di vom britischen Geheimdienst getötet wurde, auch eher denkt, dass sie ihren eigenen Tod nur vorgetäuscht habe. Es geht also weniger um die Behauptung einer konkreten Verschwörung, sondern um den Ausdruck eines Weltbilds, in dem einige wenige sich zu ihrem Vorteil und dem Nachteil der Allgemeinheit verschworen haben - wie genau ist oft nebensächlich. Obwohl diese Weltsicht auf den ersten Blick Berührungspunkte mit der Paranoia hat (und die subklinische Paranoia auch ein stabiles Korrelat ist), gibt es einen entscheidenden Unterschied: Im paranoiden Wahn ist die ganze Welt hinter der Person her, die Verschwörungsmentalität beschreibt dagegen die Überzeugung, dass einige wenige die ganze Welt hinters Licht führen und betrügen wollen (Imhoff und Lamberty 2018). 


\section{Verbreitung von Verschwö- rungserzählungen vor der Pandemie}

Der Glaube an Verschwörungen ist weder neu noch ein reines Produkt von sozialen Medien. Auch in der Gegenwart und vor der Pandemie waren solche Narrative in der Gesellschaft verbreitet: Über $40 \%$ der Befragten der Studie „Verlorene Mitte - Feindselige Zustände. Rechtsextreme Einstellungen in Deutschland 2018/19“ der Friedrich-Ebert-Stiftung waren mehr oder weniger stark der Meinung, dass „Politiker und andere Führungspersönlichkeiten nur Marionetten der dahinterstehenden Mächte" seien, so die Ergebnisse der Studie (Zick et al. 2019).

\section{Psychologische Ursachen des Verschwörungsglaubens}

Warum wittern Menschen nun aber Verschwörungen und glauben, dass die Geschicke der Welt von geheimen Mächten gelenkt werden? In der psychologischen Forschung werden dazu 3 Motive benannt (Douglas et al. 2017):

\section{Existenzielle Motive}

Existenzielle Motive sind erst einmal ganz basale Bedürfnisse des Überlebens. Es geht um Kontrolle und Sicherheit. Im Jahr 2008 veröffentlichten die Psycholog:innen Whitson und Galinsky (2008) im hochrangigen Wissenschaftsmagazin Science einen Forschungsbeitrag, in dem sie zeigten, dass, wenn Menschen einen Kontrollverlust erleben, sie eher dazu neigen, auch dort Muster zu sehen, wo (vermutlich) keine sind, und stärker an Verschwörungserzählungen glauben. Sie konnten zeigen, wie relevant und einschneidend Kontrollverlust für die Psyche ist. Das mag vielleicht erst einmal abstrakt klingen, Kontrolle spielt aber im Leben eine wichtige Rolle. Der plötzliche Verlust von Angehörigen, überraschende Arbeitslosigkeit oder der ICE, den man verpasst, weil die S-Bahn zu langsam war - das sind alles Beispiele für Kontrollverlust. Wenn Menschen objektiv keine Kontrolle herstellen können, versuchen sie es auf psychologischem Wege. Diese Befunde, dass experimen- tell induzierte Kontrolldeprivation in der Folge den Verschwörungsglauben steigert, ist allerdings empirisch nicht so reliabel, wie zunächst angenommen (Imhoff 2015; Stojanov und Halberstadt 2020). Was sich allerdings stabil zeigt, ist der korrelative Zusammenhang zwischen wahrgenommener geringer Kontrolle und der Verschwörungsmentalität. Erste Studien zeigen, dass politische Kontrollerfahrungen eine größere Rolle spielen als persönliche, die für eine subklinische Paranoia wiederum relevanter zu sein scheinen (Imhoff und Lamberty 2018).

\section{Soziale Motive}

Soziale Motive beziehen sich darauf, wie die eigene Gruppe und die eigene Person wahrgenommen werden. Wenn man vom Kontrollverlust hört, mag das vielleicht ein Bild eines unsicheren, entfremdeten "Gläubigen“ hervorrufen, der sich verzweifelt an Verschwörungen klammert, um die verwirrende und unkontrollierbare Welt zu verstehen. Viele Verschwörungsideolog:innen sind aber sehr lautstark, wenn es darum geht, ihre alternativen Wahrheiten über das Internet oder bei öffentlichen Veranstaltungen zu verbreiten. „Die Wahrheit“ zu sehen, kann nicht nur Kontrolle erzeugen, sondern auch das Gefühl verstärken, besonders zu sein. Sich von der Masse abzuheben, ist primär ein grundlegender Aspekt der menschlichen Existenz. Menschen mit einer besonders starken Motivation, sich von anderen zu unterscheiden, können allerdings besonders anfällig für Verschwörungserzählungen sein (Imhoff und Lamberty 2017). Auch kollektiver Narzissmus spielt im Verschwörungsglauben eine Rolle (Golec de Zavala und Federico 2018; Marchlewska et al. 2019). Narzissmus bedeutet ja nicht nur, dass eine Person sich für grandios hält, sondern dass dieses Selbstbild fragil und von der Bestätigung anderer abhängig ist. Beim kollektiven Narzissmus glauben Menschen auf der einen Seite an die Grandiosität ihrer eigenen Gruppe, gehen aber gleichzeitig davon aus, dass andere diese Grandiosität nicht genug wertschätzen. In dieser Logik zielt jede Kritik an der Gruppe darauf $\mathrm{ab}$, angeblich die Gruppe als Ganzes zu untergraben und $\mathrm{zu}$ bedrohen. Kollektive Narzisst:innen sehen ihre Nation oder Gruppe als Opfer, deren Großartigkeit und Einzigartigkeit nicht genug geschätzt wird. Dieses Gefühl, dass die eigene Gruppe nicht den Raum erhält, den sie angeblich verdient, führt dazu, dass negative Gefühle auf Verschwörer externalisiert werden und ihnen die Schuld am Scheitern der eigenen Gruppe zugeschoben wird. Das erklärt in dieser Logik, warum die Gruppe nicht immer so erfolgreich ist, wie sie es sein sollte, und nicht die Anerkennung erhält, die sie angeblich eigentlich verdient.

\section{Epistemische Motive}

Epistemische Motive beziehen sich auf den Umgang mit Fakten und Wissen. Verschwörungserzählungen bieten Erklärungen, die es den Menschen ermöglichen, den Glauben angesichts von Unsicherheit und Widerspruch zu bewahren. Verschwörungsgläubige finden eher Muster dort, wo (vielleicht) keine sind. Verschwörungserzählungen sind oft genau das: Es werden Punkte verbunden, die nicht zusammengehören. Der Zufall spielt keine Rolle. Auch hängt der Verschwörungsglaube mit der Wahrnehmung von Intentionalität zusammen (Brotherton und French 2015; Douglas et al. 2016). Zufällige Zahlencodes werden plötzlich zum umfassenden Beleg einer Verschwörung. In einer anderen Studienreihe konnte gezeigt werden, dass Menschen mit ausgeprägter Verschwörungsmentalität einen geringeren „Expertenbias“ zeigen: Während die wahrgenommene Glaubwürdigkeit mächtiger Quellen mit der Verschwörungsmentalität der Befragten abnahm, nahm die Glaubwürdigkeit machtloser Quellen zu. Je stärker der Verschwörungsglaube, desto weniger wurde zwischen Expert:innen und Laien differenziert (Imhoff et al. 2018).

\section{Weitere untersuchte Faktoren}

Auch andere Faktoren wurden in der psychologischen Forschung genauer untersucht. Eine Frage ist beispielsweise, welche Persönlichkeitsfaktoren im Ver- 
schwörungsglauben eine Rolle spielen. In verschiedenen Studien wurde daher auch der Zusammenhang mit den sog. Big Five im Fünf-Faktoren-Modell der Persönlichkeit untersucht. Eine Metaanalyse aus dem Jahr 2019 kam zu dem Schluss, dass es keinen signifikanten Zusammenhang des Verschwörungsglaubens mit den Persönlichkeitsfaktoren der Big Five gab, wenn die Effektgrößen aggregiert werden (Goreis und Voracek 2019).

\section{COVID-19-Pandemie und Verschwörungsglaube}

Insgesamt gibt es die Annahme, dass sich der Verschwörungsglaube in Krisenzeiten besonders zeigt. Krisen können als Kontrollverlust verstanden werden. Die eigene Lebenswirklichkeit wird fundamental infrage gestellt, es ist unklar, wie sich der Verlauf entwickelt, und wann alles wieder so wird wie vorher. Die Forschung von Leman und Cinnirella (2013) zeigte, dass Menschen bei großen Ereignissen eher dazu tendieren, auch große Ursachen zu vermuten. Gerade bei einer Pandemie hat man es mit einer abstrakten, unsichtbaren Bedrohung zu tun, die für Menschen oft schwer zu verstehen ist. Der Verschwörer scheint dagegen konkreter, fast "greifbar“ zu sein. Die Verschwörungserzählung strukturiert dann die Welt; es gibt die bösen Verschwörer und die, die scheinbar die Wahrheit sehen.

Theoretisch ist also anzunehmen, dass die Pandemie den Verschwörungsglauben noch einmal verstärkt hat. Empirische Schlüsse können aktuell allerdings noch nicht abschließend gezogen werden. Aus der vorherigen Forschung lässt sich annehmen, dass die abstrakte Verschwörungsmentalität eher weniger durch einen möglichen Kontrollverlust beeinflusst werden würde. Verstärkt würde insbesondere der spezifische Verschwörungsglaube. Um die Mechanismen genau $\mathrm{zu}$ verstehen, benötigt es allerdings noch weitere Daten. Dennoch lassen sich aus bisherigen Studien bereits erste Erkenntnisse ziehen.

Beginnend im Mai 2020 wurde durch das Team vom COVID-19 Snapshot Monitoring unter der Leitung von Cornelia Betsch Daten zur Verbreitung des Ver-

Psychotherapeut 2021 ·66:203-208 https://doi.org/10.1007/s00278-021-00498-2

(c) Springer Medizin Verlag GmbH, ein Teil von Springer Nature 2021

\section{P. Lamberty $\cdot$ R. Imhoff}

\section{Verschwörungserzählungen im Kontext der Coronapandemie}

\section{Zusammenfassung}

Der Glaube an Verschwörungen wird seit der weltweiten, durch die "CoronavirusKrankheit 2019" (COVID-19) ausgelösten Pandemie breit gesellschaftlich diskutiert: Seit dem Mai 2020 gab es in Deutschland bundesweit Demonstrationen, bei denen verschwörungsideologische Elemente immer wieder das Bild prägten. Die Zahl an verschwörungsideologischen Kanälen und Gruppen stieg während der Krise stark an. Viele Angehörige waren plötzlich auch im privaten Kontext damit konfrontiert, wie sie damit umgehen sollten, wenn ihnen nahestehende Menschen plötzlich überall Verschwörungen wittern. Um
Verschwörungserzählungen besser einordnen zu können, werden in diesem Beitrag die psychologischen Erkenntnisse vorgestellt, die zu erklären versuchen, warum Menschen an Verschwörungen glauben und welche Konsequenzen dieses Weltbild haben kann. Daraus ableitend wird darauf eingegangen, wie mit Fehlinformationen und Verschwörungserzählungen auch im therapeutischen Setting umgegangen werden kann.

Schlüsselwörter

COVID-19 · Verschwörungsmentalität .

Kontrollverlust · Narzissmus · Soziale Wahrnehmung

\section{Conspiracy narratives in the context of the coronavirus pandemic}

\section{Abstract}

Beliefs in conspiracies have been widely discussed in society since the worldwide outbreak of the pandemic triggered by coronavirus disease 2019 (COVID-19). Since May 2020 there have been demonstrations throughout Germany in which conspiracy ideology played an important role. The number of conspiracy ideology channels and groups rose during the crisis. Many family members were suddenly confronted in a private context with the question of how to deal with the fact that people close to them suddenly sensed conspiracies everywhere. To have a better understanding of conspiracy narratives this article therefore presents psychological insights that attempt to explain why people believe in conspiracies and what consequences this world view might have. Based on these findings the article discusses how misinformation and conspiracy narratives can be dealt with in a therapeutic setting.

\section{Keywords}

COVID-19 - Conspiracy mentality - Loss of control · Narcissism · Social perception schwörungsglaubens erhoben (Betsch et al. 2020). Hier wurden zwei Formen von Verschwörungserzählungen abgefragt (übernommen von Imhoff und Lamberty 2020): Corona sei ein Schwindel („Experten täuschen uns absichtlich und $\mathrm{zu}$ ihrem eigenen Vorteil, obwohl das Virus eigentlich nicht schlimmer ist als eine Grippe.") und Corona sei menschengemacht („Corona wurde absichtlich in die Welt gebracht, um die Bevölkerungszahl zu reduzieren."). Die Wissenschaftler:innen konnten zeigen, dass jeweils $17 \%$ diesen zwei gegensätzlichen Annahmen (eher) zustimmten. Es gab allerdings auch - obwohl sich die beiden Annahmen logisch ausschlossen eine Überlappung von $9 \%(r=0,55)$. Ins- gesamt kamen die Wissenschaftler:innen so auf Zustimmungswerte von $25 \%$ für die beiden Verschwörungserzählungen, die so über die nächsten Monate bis November stabil auf diesem Niveau blieben (COVID-19 Snapshot Monitoring 2020).

Dass dieser Glaube an eine große Verschwörung hinter der momentanen Pandemie keine harmlose Petitesse ist, zeigt sich nicht nur daran, dass die $\mathrm{Zu}$ stimmung mit geringerer selbstberichteter Compliance mit den empfohlenen Maßnahmen zur Infektionseindämmung korreliert ist (Hygiene und Abstand; Imhoff und Lamberty 2020) und sich dies auch längsschnittlich im Sinne eines kausalen Vorlaufens belegen lässt (Bier- 
wiaczonek et al. 2020; Pummerer et al. 2020).

Längst sind die diversen Demonstrationen von sog. Coronaleugner:innen zum Politikum geworden. Menschen, die sich ohne Abstand und Mund-NaseSchutz zu größeren Demonstrationen versammeln, aus denen heraus Sicherheitskräfte \& Pressevertreter:innen beschimpft und angegriffen werden. Und schließlich eine große Anzahl verunsicherter Angehöriger, die sich an Sektenbeauftragte und andere Stellen wenden, in der Hoffnung zu verstehen, wie sie den Spuk beenden oder zumindest weiter mit ihren Kindern, Eltern, Partnern und anderen eigentlich vertrauten Personen reden können. Zwei zentrale Herausforderungen sind also, wie man Verschwörungserzählungen in der Öffentlichkeit begegnen kann und wie man im persönlichen oder therapeutischen Setting mit ihren Anhänger:innen umgeht.

\section{Umgang mit Verschwörungs- erzählungen}

Es ist erst einmal wichtig, sich klar zu machen, dass es einen Unterschied zwischen Fehl- oder Desinformation und Verschwörungserzählungen gibt. Während Fehlinformationen leichter entlarvt werden können, ist dies bei kryptischen Mythen viel schwieriger. Wie möchte man etwas widerlegen, das es gar nicht gibt? Zusätzlich zeigt sich die Tendenz, dass Verschwörungsgläubige eher dazu neigen, gegen eine "offizielle Erklärung“ als für eine konkrete Alternative zu argumentieren (Wood und Douglas 2013). Das macht es noch einmal unwahrscheinlicher, dass Ansätze erfolgreich sind, die die Verschwörungsannahme widerlegen wollen.

Trotzdem ist es auch im therapeutischen Setting wichtig, sich mit den Gefahren sowie dem Umgang mit Fehlinformationen auseinanderzusetzen. Denn diese haben sich während der Pandemie schnell verbreitet. Immer wieder werden Berichte über Menschen veröffentlicht, die gestorben sind, nachdem sie entweder das Virus geleugnet oder Desinformationen zu Behandlungsmöglichkeiten geglaubt haben. Ein Problem: Fehlinformationen bleiben oft im Gedächtnis - selbst, wenn sie korrigiert worden sind. Erklärt wird das u.a. durch den Perseveranzeffekt. „Dieser beschreibt, dass Informationen oder Eindrücke oft so stark prägen, dass eine einmal gebildete Meinung auch durch später hinzukommende neue Informationen - oder sogar durch den expliziten Widerruf der ursprünglichen Information - kaum noch verändert werden kann“, erklären Miketta und Liepelt (2020). Eine Möglichkeit, diesem Effekt entgegenzuwirken, ist das „Fakten-Sandwich“: Zuerst beginnt man mit einem eingängigen Fakt - so simpel und konkret wie möglich. Als Nächstes warnt man, dass jetzt eine Fehlinformation kommt. Diese sollte nur einmal wiederholt werden. Danach kann man erklären, wie genau der Fehlschluss aussieht, und warum die Information irreführend ist. Als Letztes endet man wieder mit dem Fakt. Diesen kann man auch mehrfach wiederholen (Lewandowsky et al. 2020). Es gibt auch einige andere Ansätze, die sich als effektivim Umgang mit Fehlinformationen gezeigt haben. Digitale Spiele wie „Get Bad News“ oder „Go viral!“ beispielsweise helfen jungen Menschen dabei, signifikant besser darin zu werden, Fake News zu erkennen. Beide Spiele sind kostenlos nutzbar und für Kinder ab 12 Jahren geeignet.

Im Kontext von Verschwörungserzählungen empfehlen Sektenberatungsstellen, sich weniger auf die Auseinandersetzung mit Fakten als auf die individuelle Funktion des Verschwörungsglaubens zu fokussieren: Gab es Krisen, die Anhänger:innen von Verschwörungserzählungen durchlebt haben? Wurden diese Erfahrungen vielleicht auf einen Verschwörer projiziert? Auch wenn es erst einmal herausfordernd ist, mit Anhänger:innen von Verschwörungserzählungen umzugehen, ist es wichtig, sich bewusst zu machen, dass dies nur einen Teil der Identität darstellt. Das Thema sollte zwar adressiert werden, muss aber nicht permanent im Zentrum stehen, da sonst auch die Abwehrhaltung der Betroffenen verstärkt werden kann.

Gerade in der Pandemie kann der Verschwörungsglaube auch damit zusammenhängen, dass Maßnahmen nicht eingehalten werden und so das Risiko einer Ansteckung auch für andere erhöht ist.
Hier kann es helfen, persönliche Botschaften wie beispielsweise "Auch wenn Sie nicht an die Pandemie glauben, ist es mir wichtig, dass Sie in diesen Räumen den Regeln folgen“ zu senden. Die Person kann dann die AHA-Regeln, i.e. den Abstand zu halten, die Hygiene zu beachten und im Alltag eine Maske zu tragen, befolgen, ohne an diesem Punkt schon den eigenen Irrtum eingestehen $\mathrm{zu}$ müssen.

Verschwörungsideolog:innen haben verschiedene Strategien, um ihre Inhalte $\mathrm{zu}$ verbreiten. Dazu gehören beispielsweise die Verwendung emotional aufgeladener Sprache, der Einsatz von falschen Expertenaussagen und die Erschaffung von Sündenböcken. Wer diese Muster kennt, kann sie leichter erkennen und anderen erklären. Man verliert sich dann nicht in Faktendiskussionen und Nebenschauplätzen, sondern kann gemeinsam auf einer Metaebene erarbeiten, welche Muster bedient werden.

Es kann auch passieren, dass Therapeut:innen selbst als Teil der Verschwörung wahrgenommen werden. Es hilft dann meistens wenig, defensiv die Annahme auf Faktenebene zu dekonstruieren, da oft einfach neue Verschwörungsannahmen erschaffen werden. Vertrauensbildende Maßnahmen und ein Ansatz, bei dem das Gegenüber selbst die Verschwörungsannahme erklären muss, können eher hilfreich sein. Allerdings gibt es aktuell noch keine Studien, die sich mit dieser speziellen Thematik befassen. Hier ist weitere Forschung wünschenswert, damit der Verschwörungsglaube nicht zum Therapiehindernis wird.

\section{Fazit für die Praxis}

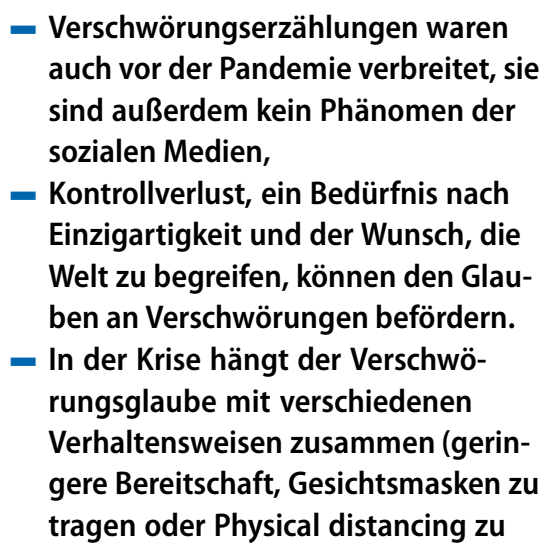


betreiben, stärkere Ablehnung von Impfungen, Zuwendung zu [teils] gefährlichen Alternativen) und kann damit ein Risiko beim Management der Pandemie sein.

- Das Fakten-Sandwich kann dabei helfen, Fehlinformationen zu entkräften.

- Beim individuellen Umgang mit Menschen, die einen starken Verschwörungsglauben aufweisen, wird von Beratungsstellen empfohlen, die Selbstwirksamkeit zu stärken und auf die Motive hinter dem Verschwörungsglauben abzuzielen.

\section{Korrespondenzadresse}

Pia Lamberty, M.Sc.

Sozial- und Rechtspsychologie, JohannesGutenberg-Universität Mainz Binger Str. 14-16, 55122 Mainz, Deutschland pia.lamberty@uni-mainz.de

\section{Einhaltung ethischer Richtlinien}

Interessenkonflikt. P. Lamberty und R. Imhoff geben an, dass kein Interessenkonflikt besteht.

Für diesen Beitrag wurden von den Autoren keine Studien an Menschen oder Tieren durchgeführt. Für die aufgeführten Studien gelten die jeweils dort angegebenen ethischen Richtlinien.

\section{Literatur}

Betsch C, Korn L, Felgendreff L, Eitze S, Schmid P, Sprengholz P, Wieler L, Schmich P, Stollorz V, Ramharter M, Bosnjak M, Omer SB, Thaiss H, De Bock F, Von Rüden U, Imhoff R (2020) German COVID-19SnapshotMonitoring (COSMO)-Welle 10 (05.05.2020). PsychArchives. https://doi.org/ 10.23668/PSYCHARCHIVES.2900 Accessed: 12 Feb 2021.

Bierwiaczonek K, Kunst JR, Pich O (2020) Belief in COVID-19 conspiracy theories reduces social distancing over time. Appl Psychol Health Well Being 12:1270-1285

Brotherton R, French CC (2015) Intention seekers: Conspiracist ideation and biased attributions of intentionality. PLoSONE 10:e124125

COVID-19 Snapshot Monitoring Verschwörungsdenken. COVID-19 Snapshot Monitoring. https://projekte.uni-erfurt.de/cosmo2020/ web/topic/vertrauen-ablehnung-demos/30verschwoerung/ (Erstellt: 20. Nov. 2020). Zugegriffen: 6. Jan. 2021

Douglas KM, Sutton RM, Callan MJ, Dawtry RJ, Harvey AJ (2016) Someone is pulling the strings: hypersensitive agency detection and belief in conspiracy theories. Think Reason 22:57-77

Douglas KM, Sutton RM, Cichocka A (2017) The psychology of conspiracy theories. Curr Dir Psychol Sci 26:538-542
Goertzel T (1994) Belief in conspiracy theories. Polit Psychol. https://doi.org/10.2307/3791630

Golec de Zavala A, Federico CM (2018) Collective narcissism and the growth of conspiracy thinking over the course of the 2016 United States presidential election: A longitudinal analysis. Eur J Soc Psychol 48:1011-1018

Goreis A, Voracek M (2019) A systematic review and meta-analysis of psychological research on conspiracy beliefs: field characteristics, measurement instruments, and associations with personality traits. Front Psychol 10:205

ImhoffR (2015) Beyond (right-wing) authoritarianism. In: Bilewicz M, Cichocka A, Soral W (Hrsg) The psychology of conspiracy. Taylor \& Francis, New York, S 122-142

Imhoff R, Bruder M (2014) Speaking (un-) truth to power: conspiracy mentality as a generalised political attitude. Eur J Pers 28:25-43

Imhoff R, Lamberty P (2018) How paranoid are conspiracy believers? Toward a more finegrained understanding of the connect and disconnect between paranoia and belief in conspiracy theories. Eur J Soc Psychol 48:909-926

Imhoff R, Lamberty P (2020) A bioweapon or a hoax? The link between distinct conspiracy beliefs about the Coronavirus disease (COVID-19) outbreak and pandemic behavior. Soc Psychol Personal Sci 11:1110-1118

Imhoff R, Lamberty PK (2017) Too special to be duped: Need for uniqueness motivates conspiracy beliefs. Eur J Soc Psychol 47:724-734

Imhoff R, Dieterle L, Lamberty P (2021) Resolving the puzzle of conspiracy worldview and political activism: belief in secret plots decreases normative but increases nonnormative political engagement. Soc Psychol Personal Sci 12:71-79

Imhoff R, Lamberty P, Klein O (2018) Using power as a negative cue: how conspiracy mentality affects epistemic trust in sources of historical knowledge. Pers Soc Psychol Bull 44:1364-1379

Leman PJ, Cinnirella M (2013) Beliefs in conspiracy theories and the need for cognitive closure. Front Psychol 4:378

Lewandowsky S, Cook J, Ecker UK, Lewandowsky S, Cook J, Ecker UKH, Newman EJ (2020) Under the Hood of The Debunking Handbook 2020: A consensus-based handbook of recommendations for correcting or preventing misinformation. https:// www.climatechangecommunication.org/wpcontent/uploads/2020/10/DebunkingHand book2020.pdf.Zugegriffen:6. Jan. 2021

Marchlewska M, Cichocka A, Łozowski F, Górska P, Winiewski M (2019) In search of an imaginary enemy: Catholic collective narcissism and the endorsement of gender conspiracy beliefs. J Soc Psychol 159:766-779

Miketta S, Liepelt R (2020) Kein Weg zurück: Warum Fake News über Corona hängenbleiben, selbst wenn wir wissen, dass sie falsch sind. In-Mind. https://de.in-mind.org/blog/ post/kein-weg-zurueck-warum-fake-newsueber-corona-haengenbleiben-selbst-wennwir-wissen-dass. Zugegriffen:6. Jan. 2021

Pummerer L, Böhm R, Lilleholt L, Winter K, Zettler $\mathrm{I}$, Sassenberg K Conspiracy theories and their societal effects during the COVID-19 pandemic. https://doi.org/10.31234/osf.io/y5grn (Erstellt: 14. Apr. 2020). Accessed: 12 Feb 2021.

Stojanov A, Halberstadt J (2020) Does lack of control lead to conspiracy beliefs? A meta-analysis. Eur J Soc Psychol 50:955-968
Whitson JA, Galinsky AD (2008) Lacking control increases illusory pattern perception. Science 322:115-117

WHO (2020) Managing the COVID-19 infodemic: Promoting healthy behaviours and mitigating the harm from misinformation and disinformation. https://www.who. int/news/item/23-09-2020-managing-thecovid-19-infodemic-promoting-healthybehaviours-and-mitigating-the-harm-frommisinformation-and-disinformation. Zugegriffen: 6. Jan. 2021

Wood MJ, Douglas KM (2013) “What about building 7?" A social psychological study of online discussion of 9/11 conspiracy theories. Front Psychol 4:409

Wood MJ, Douglas KM, Sutton RM (2012) Dead and alive: beliefs in contradictory conspiracy theories. Soc Psychol Personal Sci 3:767-773

Zick A, Küpper B, Berghan W, Schröter F, Faulbaum F, Häusler A, Rees JH (2019) Verlorene Mitte-Feindselige Zustände: Rechtsextreme Einstellungen in Deutschland 2018/19. Dietz, Berlin 
Hier steht eine Anzeige.

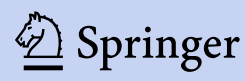

